\title{
Mise en évidence des domaines sur les ferrites doux par effet Kerr longitudinal
}

\author{
R. KACZMAREK, M. DAUTAIN et T. BARRADI-ISMAIIL
}

Service Electrotechnique et Electronique Industrielle, Ecole Superieure d'Electricite, 91192 Gif sur Yvette, France

\begin{abstract}
For the first time, the longitudinal Kerr effect has been used in order to observe magnetic domains and their development in power ferrites. Image subtraction and processing leads to a magnetic contrast being a quasi derivative of the domains. A kind of integration procedure applied to them permits a reconstruction of a local hysteresis which parameters closely approach the global hysteresis data.
\end{abstract}

\section{Introduction}

La minimisation de la taille des convertisseurs DC-DC se heurte aux problèmes d'échauffement dûs aux pertes dans les circuits magnétiques des transformateurs. Pour comprendre le mécanisme des pertes, l'analyse des mouvements des domaines est nécessaire. Or, l'observation des domaines dans les ferrites doux et isotropes, qui composent le noyau des transformateurs haute fréquence, était jusqu'à présent limitée par manque de méthodes efficaces. La méthode de BITTER, utilisée dans les ferrites depuis déjà bien longtemps $(1,2)$ s'applique en statique seulement, à basse température et essentiellement à des matériaux anisotropes. L'effet KERR (3) est dépourvu des inconvénients de la méthode de BITTER, mais est difficile à mettre en oeuvre sur les ferrites de puissance. Des travaux antérieurs ont été effectués sur des ferrites anisotropes où l'effet polaire, relativement fort, a pu être utilisé ou encore sur des monocristaux à saturation élevée, où l'effet Kerr longitudinal s'avère adapté.

La configuration longitudinale est essentielle dans le cas de tores de ferrites polycristallins et isotropes, mais les ferrites de puissance ont des aimantations à saturation plus petites que les monocristaux $(0.4 \mathrm{~T}$ et $0.6 \mathrm{~T}$ respectivement). La rotation de la polarisation est donc plus faible que sur les monocristaux où elle est de l'ordre d'une minute. D'autre part la petite taille des grains (quelques dizaines de microns) impose un grossissement fort, ce qui réduit encore plus le contraste. Ces différentes contraintes expliquent l'absence de résultats antérieurs pour ce type de matériaux.

Cet article présente une méthode d'observation et d'analyse des domaines dans les ferrites de puissance. 


\section{Observation des domaines}

Pour que le très faible contraste de l'effet longitudinal sur les ferrites doux polycristallins puisse se manifester, il faut soigner particulièrement la source de lumière et les algorithmes de traitement d'images. Sur notre banc d'essai (cf. Fig. 1), le laser assure une puissance variable de la lumière, la fibre monomode permet une répartition quasi gaussienne de la tache lumineuse et le stabilisateur de lumière filtre les variations basse fréquence $(<5 \mathrm{kHz})$.

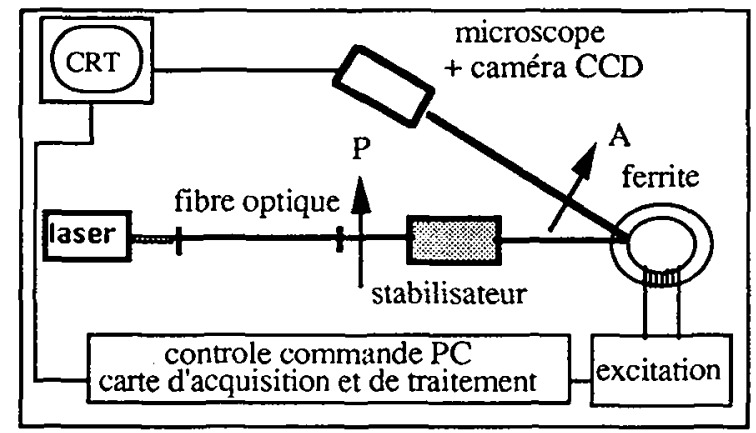

FIG. 1 : Banc d'essai, $\mathrm{P}$ - polariseur, A - analyseur

L'image acquise par une caméra $\mathrm{CCD}$ à gain et offset variables, est numérisée et traitée à l'aide d'algorithmes comprenant moyennage, soustraction avec offset, filtrage, redistribution des niveaux de gris et "zoom". Sur une image avant soustraction, dont l'histogramme s'étale entre 0 et 255 , l'information magnétique se situe sur quelques niveaux, le plus souvent 3 ou 4 niveaux de gris. Ainsi, il n'est pas possible de visualiser les domaines directement. La seule soustraction de deux images à excitation différente n'est pas suffisante pour révéler un contraste magnétique. Le moyennage élimine le bruit non corrélé, la soustraction supprime le contraste non magnétique, le filtrage traite différents types de perturbations, comme le bruit haute fréquence par exemple. Ainsi le contraste magnétique sur les ferrites doux polycristallins a pu être mis en évidence ; la figure 2 présente un exemple.

Cette photo montre le contraste résultant de la soustraction de deux images à excitations différentes. En fait, pour les contrastes très faibles, seule la quasi dérivée de l'image magnétique peut être visualisée $(4,5)$. Sur la Fig. 2, la première des deux images soustraites ayant subi un "offset" de 6 , nous avons mesuré une valeur moyenne de 4.1 pour le noir, 6.6 pour le gris et 9.0 pour le blanc. Cette exemple montre que sur les ferrites de puissance nous sommes amenés à opérer à la limite de la résolution numérique. 


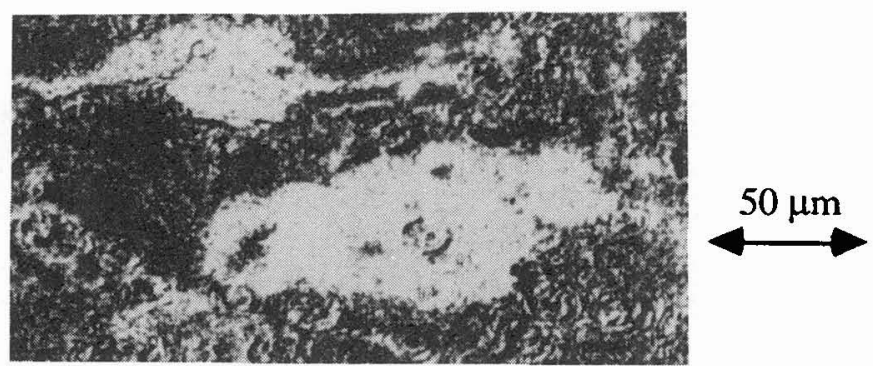

FIG. 2 : Exemple de structure de domaines diférentiels sur les ferrites de puissance

\section{Analyse du contraste magnétique}

Avec la polarisation normale au plan d'incidence, seule la composante longitudinale de l'aimantation $\mathrm{M}_{1}$ intervient comme cause de la rotation de la polarisation. Entre la saturation négative et positive, cette composante varie entre deux valeurs maximales et opposées. Avec l'analyseur et le polariseur légèrement décroisés, sous l'action d'une excitation monotone (9), si l'aimantation tourne toujours dans un même sens, alors l'intensité de la lumière polarisée à la sortie de l'analyseur est monotone (Fig. 3).

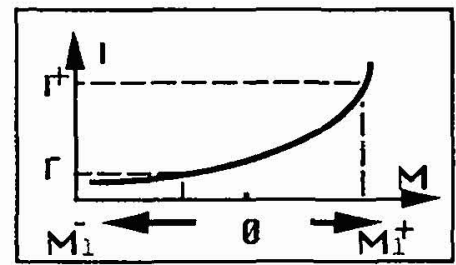

FIG. 3 : Intensité à la sortie de l'analyseur. $\mathrm{M}_{1}^{-}$et $\mathrm{M}_{1}^{+}$sont les composantes longitudinales à saturation négative et à saturation positive respectivement. L'analyseur et le polariseur sont légèrement décroisés.

Dans ces conditions la différence de deux images à l'excitation différente présentera un contraste toujours noir (ou blanc) sur toute la plage d'excitation. Il est donc possible de numériser le contraste et d'estimer avec une bonne précision le caractère hystérétique des phénomènes magnétiques sur la surface des ferrites.

\section{Hystérésis}

L'évaluation de l'hystérésis locale par quantification des variations des domaines est pratiquée depuis longtemps $(6,7,8)$, mais les méthodes utilisées étaient appliquées dans le cas de domaines visibles directement sans traitement spécial. Dans le cas des images différentielles nous avons affaire à des quasi dérivées des domaines. La reconstitution des domaines d'origine passe par une quasi intégration du contraste magnétique. Ainsi on obtient une mesure de l'induction locale, qui peut-être représentée sur un graphe d'hystérésis locale.

Nous avons acquis 17 images qui correspondent aux différentes excitations le long de la courbe d'hystérésis. Avec la soustraction récurrente, cela donne 16 images différentielles qui révèlent une évolution du contraste 
magnétique (Fig.4). Le contraste noir correspond au passage de la saturation négative à la saturation positive, le contraste blanc représente le retour.
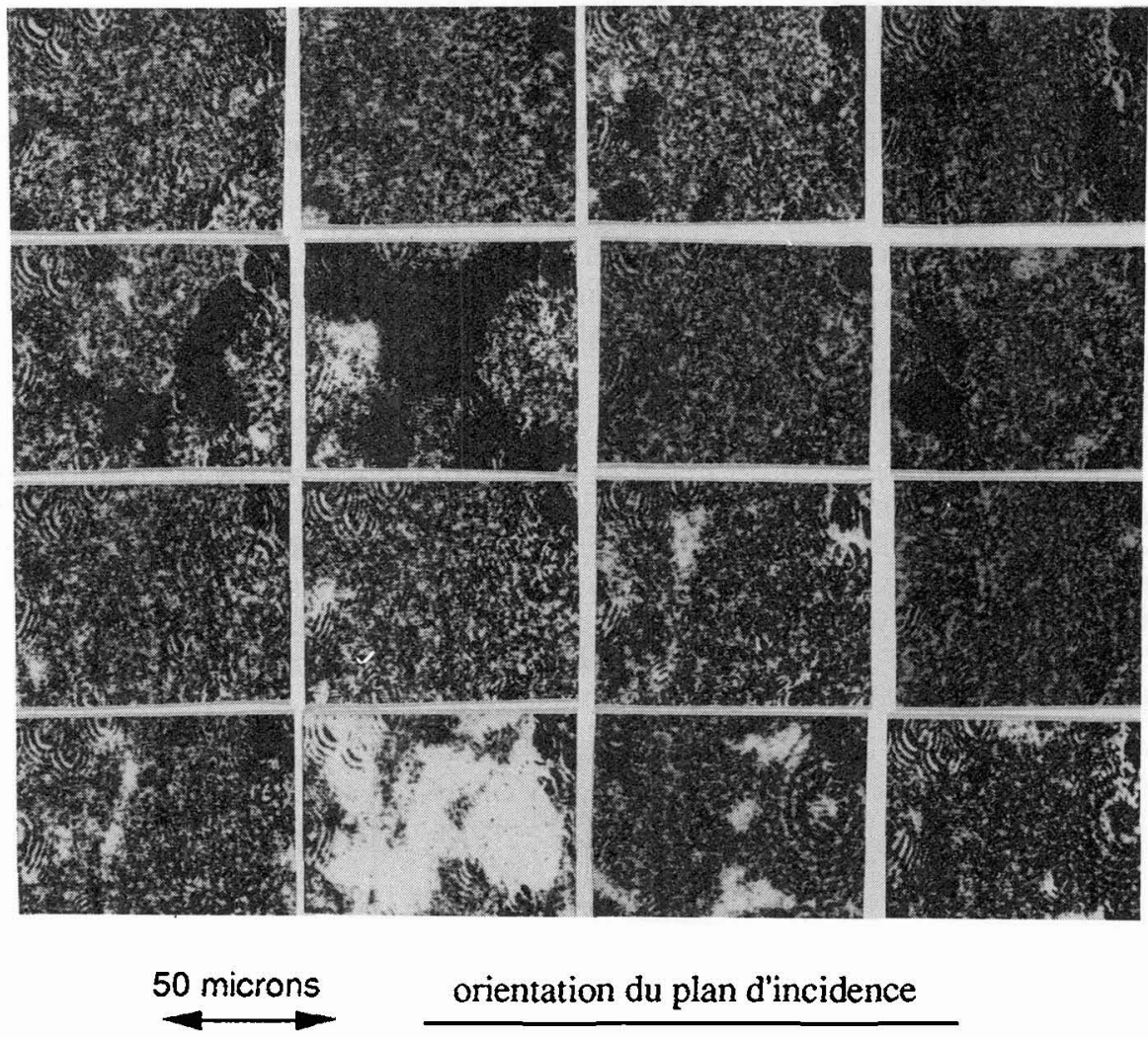

FIG 4 : Contraste magnétique différentiel le long de la courbe d'hystérésis. Le plan d'incidence est perpendiculaire à la surface des images.

En mesurant les surfaces des zones de contraste magnétique, nous obtenons la courbe d'hystérésis locale (ligne continue sur la Fig.5), laquelle présente une assez bonne ressemblance avec la courbe d'hystérésis globale relevée de façon macroscopique (ligne discontinue sur la Fig. 5).

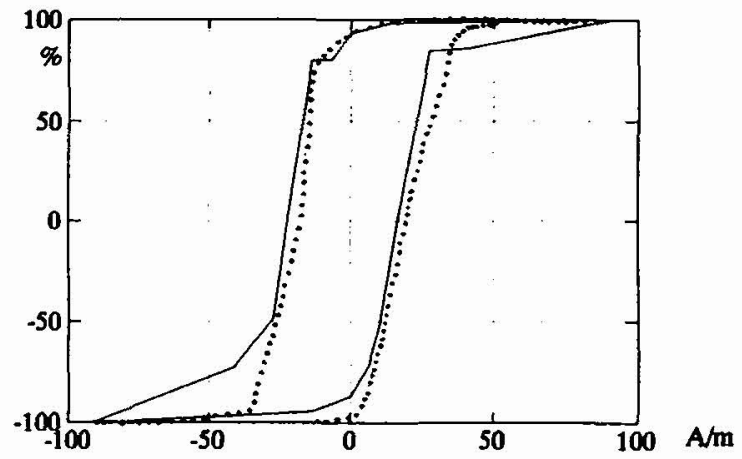

FIG. 5 : Hystérésis local et global sur un ferrite de puissance. 
Les valeurs caractéristiques globales obtenues à $1 \mathrm{kHz}$ sont les suivantes :

$\mathrm{H}_{\mathrm{C}}=17 \mathrm{Am}^{-1}, \mathrm{~B}_{\text {sat }}=0.351 \mathrm{~T}, \mathrm{~B}_{\mathrm{rem}}=0.326 \mathrm{~T}=93 \% \mathrm{~B}_{\text {sat }}$.

Les mêmes paramètres obtenus localement sont très similaires :

$\mathrm{H}_{\mathrm{C}^{+}}=16 \mathrm{Am}^{-1}, \mathrm{H}_{\mathrm{C}^{-}}=-22 \mathrm{Am}^{-1}, \mathrm{M}_{\mathrm{rem}}{ }^{+}=93 \% \mathrm{M}_{\mathrm{sat}}, \mathrm{M}_{\mathrm{rem}}^{-}=-87 \% \mathrm{M}_{\text {sat }}$.

\section{Conclusions}

A l'aide d'un dispositif bien soigné et d'algorithmes performants de traitement d'images, nous avons réussi à visualiser les domaines sur les ferrites de puissance. L'analyse des domaines différentiels ainsi obtenus permet de trouver des valeurs des paramètres hystérétiques locaux proches des valeurs obtenues par les mesures de l'hystérésis globales.

Les améliorations expérimentales sont envisagées sur deux points :

- l'optimisation des performance du matériel, comme l'adaptation des paramètres de la caméra (facteur gamma proche de 1 , numerisation du signal vidéo sur 12 voire 16 bits)

- l'amplification de la rotation Kerr sur les ferrites de puissance par des méthodes qui restent à développer.

Les prochains travaux doivent mettre en évidence des éléments de corrélation entre le contraste magnétique et la microstructure du matériel comme l'anisotropie locale, les rotations pures de l'aimantation et les déplacements de parois, la transition au caractère monodomaine des grains en fonction de leur taille.

\section{Remerciements}

Nous remercions le Ministère de la Recherche et de la Technologie pour son soutien financier concernant ce travail.

\section{Bibliographie}

1/ Bates L.F., Craik D.J., Griffiths P.M. - Proc. Phys. Soc. vol. 71, part 5, 1958

2/ Tebble R.S., Williams D.E.G. - brit. Journ. Appl. Phys. vol. 11, 1960

3/Fowler C.A., Fryer E.M., Brandt B.L., Isaacson R.A. - Journ. Appl. Phys. vol. 34, nr. 4, 1963

4/ Schmidt F., Rave W., Hubert A. - IEEE Trans. Magn. MAG-21, 1985

5/ Argyle B.E., Petek B., Herman D.,A. - Journ. Appl. Phys. 61, 1987

6/ Knowles J.E. Proc. Phys. Soc LXXV, 6 (1959)

7/ Beckley P., Thompson J.E. Proc. IEE vol 117, nr. 11 (1970)

8/ Mivenchi E., Beckley P., HorrocksD.H. - SMM'10, 1990

9/ Kaczmarek R.,Dautain M.,Barradi T. - Proceedings of IFC 6, Tokyo 1992 